\title{
Simulação Numérica Aplicada para Avaliar o Efeito da Pré-polimerização no Comportamento de Reatores Tubulares
}

\author{
André L. Nogueira, Ricardo A. F. Machado, Marintho B. Quadri \\ Departamento de Engenharia Química, UFSC
}

\author{
Liliane M. F. Lona \\ Faculdade de Engenharia Química, DPQ, UNICAMP
}

Resumo: O presente estudo utiliza um modelo matemático fenomenológico para simular um sistema de polimerização contínuo em dois estágios. Este sistema é composto por um reator contínuo tipo tanque agitado (CSTR), para pré-polimerização do monômero (primeiro estágio), associado em série a um reator tubular para conduzir a reação até elevados valores de conversão (segundo estágio). Um modelo detalhado, considerando variações axiais e radiais, assim como operação não-isotérmica, foi utilizado para simular o comportamento do reator tubular em diferentes situações. Um modelo de caracterização também foi desenvolvido para fornecer estimativas do peso molecular médio e do índice de polidispersão do polímero. Os resultados mostram que reações de polimerização conduzidas em sistemas contínuos de dois estágios fornecem um polímero com propriedades menos heterogêneas do que um polímero obtido em um sistema reacional composto por apenas um reator tubular. Além disso, quanto maior a viscosidade da mistura reacional alimentada ao reator tubular, mais homogêneo é o polímero obtido.

Palavras-chave: Pré-polimerização, CSTR, reator tubular, modelagem, polimerização em solução, estireno.

\section{Numerical Simulation to Evaluate the Effect from Pre-Polymerization on the Behavior of Tubular Reactors}

Abstract: The present study uses a phenomenological model to simulate a continuous, two-stage polymerization process. This system is composed by a continuous stirred tank reactor (CSTR) for monomer pre-polymerization (first stage), connected to a tubular reactor (second stage) to carry out the reaction up to high conversion values. A comprehensive non-isothermal 2-D model (axial and radial variations) was used to predict the tubular reactor behavior. A polymer characterization model was also developed to provide estimates of the polymer average molecular weight and polydispersity. According to the results, polymerization reactions carried out in a continuous two-stage system provide a polymer with less heterogeneous properties than the one obtained in a single tubular reactor. Besides, it is possible to produce a more homogeneous polymer increasing the viscosity of the mixture fed in the tubular reactor.

Keywords: Pre-polymerization, CSTR, tubular reactor, modeling, solution polymerization, styrene.

\section{Introdução}

A elevada e crescente competitividade de mercado entre indústrias fazem com que estas busquem constantemente por condições operacionais que maximizem a capacidade produtiva da planta instalada e minimizem seus custos de operação, mantendo os níveis de segurança operacional e da qualidade do produto. Neste cenário, os simuladores de processo aparecem como uma ferramenta muito útil e viável. Eles são capazes de explorar determinado processo e fornecer boas estimativas de condições operacionais ótimas para atingir objetivos específicos, sem custos referentes a reagentes e a ocupação da instalação industrial, além de não oferecerem riscos de segurança. Os simuladores também oferecem a vantagem de facilitar a compreensão sobre o relacionamento entre as variáveis de processo e os parâmetros de qualidade do produto, podendo ainda ser utilizados no treinamento de operadores em situações de risco, partida e parada de plantas industriais.

Industrialmente, reações de polimerização realizadas em CSTR's não são conduzidas até altos valores de conversão devido ao considerável aumento da viscosidade da mistura reacional, o que dificulta a agitação e a transferência de calor, aumentando o custo operacional e fornecendo um polímero com propriedades heterogêneas ${ }^{[1]}$. Ao contrário, os reatores tubulares são economicamente atrativos, pois sua simplicidade geométrica leva a baixos custos fixos e operacionais. A grande área superficial de troca térmica do tubo é particularmente vantajosa para reações de polimerização, já que estas são fortemente exotérmicas.

Apesar das vantagens que os reatores tubulares apresentam, eles não são muito utilizados na produção comercial de polímeros devido a elevada heterogeneidade dos produtos

Autor para correspondência: André L. Nogueira, Departamento de Engenharia Química, UFSC, Campus Universitário - Trindade, CEP: 88040-900, Florianópolis, SC, Brasil. E-mail: andren@enq.ufsc.br 
obtidos nestes equipamentos. Isto se deve ao complexo comportamento reológico da solução polimérica ao longo destes reatores (largos gradientes radiais) e ao resultante alargamento da distribuição dos tempos de residência.

A literatura apresenta alguns trabalhos, experimentais e/ou de simulação, que empregam alternativas operacionais capazes de auxiliar na redução destes gradientes. Cabral et al. ${ }^{[2]}$ utilizam um reator tubular vazio operando com reciclo para avaliar o desempenho deste equipamento em função da taxa de reciclo. Os autores afirmam que a recirculação de material já polimerizado pelo reator auxilia na homogeneização das distorções dos perfis radiais da velocidade axial de escoamento.

Tien et al ${ }^{[3]}$ empregam um reator tubular com misturadores estáticos operando com altas taxas de reciclo (pré-polimerizador), associado em série a um reator tubular com misturadores estáticos e sem reciclo, para comparar o comportamento do sistema operando com e sem pré-polimerização. Apesar da utilização de um pré-polimerizador, o estudo não aborda os efeitos do uso de um primeiro estágio nos perfis de velocidade desenvolvidos no reator tubular. Porém, afirma que o uso de misturadores estáticos é capaz de superar as problemáticas de heterogeneidade do produto.

Apesar de $\mathrm{Chen}^{[4]}$ também utilizar um sistema de polimerização em dois estágios (CSTR associado em série a um reator tubular), nenhum estudo é realizado com relação aos efeitos da pré-polimerização sobre os perfis radiais no reator tubular, uma vez que misturadores estáticos são utilizados neste equipamento. Já em Lynn e Huff ${ }^{[5]}$, a utilização de um pré-polimerizador antes do reator tubular apenas é sugerida como alternativa de projeto para reduzir os fortes perfis radiais desenvolvidos ao longo de reatores tubulares vazios.

Em Husain e Hamielec ${ }^{[6]}$, os autores apenas reportam que o uso de um CSTR como pré-polimerzador gera perfis de temperatura mais uniformes em um reator tubular. Afirmam ainda que isto se deve à eficiente dissipação de calor verificada ainda no primeiro estágio do sistema reacional.

Já o estudo realizado por Nogueira et al. ${ }^{[7]}$, um modelo bidimensional detalhado é empregado para avaliar o efeito da pré-polimerização no comportamento de reatores tubulares. O estudo compara dois sistemas reacionais de mesmo volume, sendo o primeiro um tubo vazio de volume $\mathrm{V}_{\mathrm{t}}$, e o segundo um CSTR $\left(\mathrm{V}_{\text {CSTR }}=0,2 \mathrm{~V}_{\mathrm{t}}\right)$ associado em série a um reator tubular $\left(\mathrm{V}_{\text {tubular }}=0,8 \mathrm{~V}_{\mathrm{t}}\right)$. Os resultados mostram que o uso do CSTR reduz significativamente as distorções radiais dos perfis de temperatura e velocidade axial de escoamento. Consequentemente, os perfis radiais de todas as outras variáveis de processo sofrem uma significativa suavização quando a pré-polimerização é considerada.

O presente trabalho utiliza o mesmo modelo matemático empregado por Nogueira et al. ${ }^{[7]}$ para simular o comportamento de um sistema reacional de polimerização em dois estágios (CSTR associado em série a um reator tubular). Porém, no presente estudo, esta ferramenta computacional é utilizada especificamente para aprofundar o conhecimento do efeito da pré-polimerização sobre o comportamento de reatores tubu- lares e as características finais do polímero produzido. Para isso, diferentes frações de polímero morto foram testadas na alimentação do reator tubular.

\section{Sistema Reacional e Mecanismo Cinético}

O sistema reacional contínuo considerado neste estudo compreende um reator tipo tanque agitado associado em série a um reator tubular. $\mathrm{O}$ primeiro estágio tem a finalidade de realizar a pré-polimerização do monômero, conduzindo a reação até, no máximo, valores intermediários de conversão (30-40\%). O segundo estágio é responsável por conduzir a reação até elevados valores de conversão. O estudo considera a reação de polimerização do estireno em solução de tolueno, iniciada quimicamente pelo AIBN (2,2 azo-bisisobutironitrila), devido a grande disponibilidade de dados cinéticos referentes a esta reação na literatura ${ }^{[3,8]}$. A Tabela 1 reúne os parâmetros físico-químicos utilizados nas simulações. A correlação proposta por Marten e Hamielec ${ }^{[9]}$ foi utilizada para expressar a manifestação do efeito gel.

$\mathrm{O}$ mecanismo cinético adotado considera as seguintes etapas reacionais:

- Iniciação química ${ }^{[8]}$

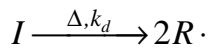

$$
\begin{aligned}
& R \cdot+M \stackrel{k_{i}}{\longrightarrow} P_{1} . \\
& {\left[k_{d}=1,0533 \cdot 10^{15} \exp \left(\frac{-15488,33}{T}\right)\right]}
\end{aligned}
$$

- Iniciação térmica ${ }^{[15]}$

$$
\begin{aligned}
& 3 M \stackrel{\Delta, k_{i t}}{\longrightarrow} 2 P_{1} . \\
& {\left[k_{i t}=1,99 \cdot 10^{6} \exp \left(\frac{-14842}{T}\right)\right]}
\end{aligned}
$$

- Propagação $0^{[16]}$

$$
\begin{aligned}
& P_{n} \cdot+M \stackrel{k_{p}}{\longrightarrow} P_{n+1} . \\
& {\left[k_{p}=1,051 \cdot 10^{7} \exp \left(\frac{-3577}{T}\right)\right]}
\end{aligned}
$$

- Transferência de cadeia para o monômero ${ }^{[17]}$

$$
\begin{aligned}
& P_{n} \cdot+M \stackrel{k_{t r m}}{\longrightarrow} P_{n}+M . \\
& M \cdot+M \stackrel{k_{t r m}}{\longrightarrow} P_{1} . \\
& {\left[k_{t r m}=2,31 \cdot 10^{6} \exp \left(\frac{-6377}{T}\right)\right]}
\end{aligned}
$$


Tabela 1. Parâmetros físico-químicos considerados nas simulações.

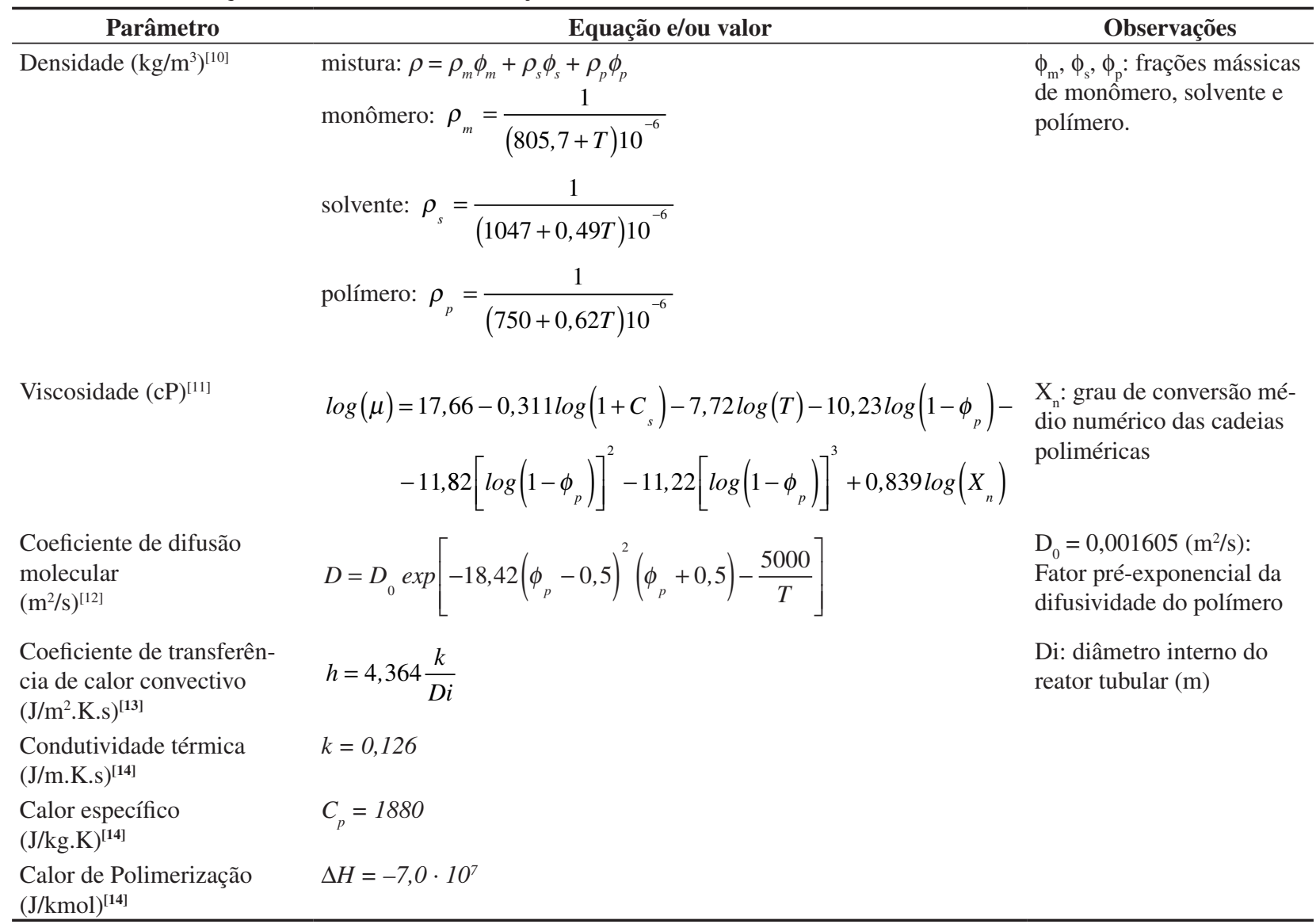

- Transferência de cadeia para o solvente ${ }^{[18]}$

$P_{n} \cdot+S \stackrel{k_{t r s}}{\longrightarrow} P_{n}+S$

$S \cdot+M \stackrel{k_{t s^{\prime}}}{\longrightarrow} P_{1}$.

$\left[k_{t r s}=5,0 \cdot 10^{-5} k_{p}\right]$

- Terminação por combinação ${ }^{[16]}$

$$
\begin{aligned}
& P_{n} \cdot+P_{m} \cdot \stackrel{k_{t c}}{\longrightarrow} P_{n+m} \\
& {\left[k_{t c}=1,255 \cdot 10^{9} \exp \left(\frac{-844}{T}\right)\right]}
\end{aligned}
$$

onde $I$ - iniciador; $M$ - monômero; $M$. - monômero ativo; $S$ - solvente; $S$. - solvente ativo; $R$. - radical livre; $P_{n}$ - polímero em crescimento com 'n' unidades monoméricas; $P_{n+m}$ - polímero desativado com ' $n+m$ ' unidades monoméricas; $k_{d}$ - constante cinética de decomposição do iniciador $\left(\mathrm{s}^{-1}\right)$; $k_{i}$ - constante cinética de iniciação química $\left(\mathrm{m}^{3} / \mathrm{kmol} . \mathrm{s}\right) ; k_{i t}$ - constante cinética de iniciação térmica $\left(\mathrm{m}^{6} / \mathrm{kmol}^{2} . \mathrm{s}\right) ; k_{p}$ constante cinética de propagação $\left(\mathrm{m}^{3} / \mathrm{kmol} . \mathrm{s}\right) ; k_{t c}$ - constante cinética de terminação por combinação $\left(\mathrm{m}^{3} / \mathrm{kmol} . \mathrm{s}\right) ; k_{t r m}$ - constante cinética de transferência de cadeia para o monômero $\left(\mathrm{m}^{3} / \mathrm{kmol} . \mathrm{s}\right)$; e $k_{t r s}$ - constante cinética de transferência de cadeia para o solvente $\left(\mathrm{m}^{3} / \mathrm{kmol}\right.$.s $)$.

\section{Modelagem Matemática e Resolução Numérica}

O modelo matemático desenvolvido para o CSTR (pré-polimerizador) é simples e considera as hipóteses de operação em estado estacionário, sistema isotérmico e mistura perfeita. Em virtude da consideração de operação em estado estacionário, um sistema composto por nove equações algébricas não-lineares foi obtido. Este modelo estacionário foi resolvido numericamente aplicando-se o Método de Newton para resolução de sistemas de equações algébricas lineares e não lineares.

Para o reator tubular, um modelo matemático detalhado foi desenvolvido, considerando a evolução dos gradientes axiais (coordenada $\mathrm{Z}$ ) e radiais (coordenada $\mathrm{r}$ ) ao longo do equipamento. Este modelo realístico assume que a velocidade axial de escoamento varia com a posição radial, produzindo perfis axiais e radiais para todas as variáveis de processo no interior do reator tubular. O modelo considera as hipóteses de operação em estado estacionário, sistema não-isotérmico, difusão radial de calor e massa, fluido Newtoniano incompressível e difusão molecular de acordo com a lei de Fick. A dissipação viscosa de calor, a presença de gradientes de pressão na direção radial, de velocidades radiais, bem como a variação dos parâmetros na direção angular, não são levadas em consideração no modelo bidimensional desenvolvido. Partindo da equação da continuidade e considerando as devidas hipóteses, obtiveram-se as seguintes equações para os 
balanços das concentrações de monômero $\left(\mathrm{C}_{\mathrm{M}}-\mathrm{mol} / \mathrm{l}\right)$, iniciador $\left(C_{\mathrm{I}}-\mathrm{mol} / \mathrm{l}\right)$ e solvente $\left(\mathrm{C}_{\mathrm{S}}-\mathrm{mol} / \mathrm{l}\right)$, além dos balanços para os momentos do polímero morto de ordem zero $\left(\mu_{0}\right)$, um $\left(\mu_{1}\right)$ e dois $\left(\mu_{2}\right)^{[5,14,19,20]}$ :

$$
\begin{aligned}
& \frac{\partial C_{M}}{\partial Z}=\left[D\left(\frac{\partial^{2} C_{M}}{\partial r^{2}}+\frac{1}{r} \frac{\partial C_{M}}{\partial r}\right)-\left(2 f k_{d} C_{I}+\right.\right. \\
& \left.\left.k_{p}[P \cdot] C_{M}+3 k_{i t} C_{M}^{3}+2 k_{t r m}[P \cdot] C_{M}+k_{t r s}[P \cdot] C_{S}\right)\right] \frac{1}{V z} \\
& \frac{\partial C_{I}}{\partial Z}=\left[D\left(\frac{\partial^{2} C_{I}}{\partial r_{2}}+\frac{1}{r} \frac{\partial C_{I}}{\partial r}\right)-k_{d} C_{I}\right] \frac{1}{V z} \\
& \frac{\partial C_{S}}{\partial Z}=\left[D\left(\frac{\partial^{2} C_{S}}{\partial r^{2}}+\frac{1}{r} \frac{\partial C_{S}}{\partial r}\right)-k_{t r s} C_{S}[P \cdot]\right] \frac{1}{V z} \\
& \frac{\partial \mu_{0}}{\partial Z}=\left[D\left(\frac{\partial^{2} \mu_{0}}{\partial r^{2}}+\frac{1}{r} \frac{\partial \mu_{0}}{\partial r}\right)+\right. \\
& \left.k_{t r m} C_{M} \lambda_{0}+k_{t r s} C_{S} \lambda_{0}+\frac{1}{2} k_{t c} \lambda_{0}^{2}\right] \frac{1}{V z} \\
& \frac{\partial \mu_{1}}{\partial Z}=\left[D\left(\frac{\partial^{2} \mu_{1}}{\partial r^{2}}+\frac{1}{r} \frac{\partial \mu_{I}}{\partial r}\right)+\right. \\
& \left.k_{t r m} C_{M} \lambda_{2}+k_{t r s} C_{S} \lambda_{2}+k_{t c}\left(\lambda_{0} \lambda_{2}+\lambda_{1}^{2}\right)\right] \frac{1}{V z} \\
& \left.k_{M} \lambda_{I}+k_{t r s} C_{S} \lambda_{I}+k_{t c} \lambda_{0} \lambda_{I}\right] \frac{1}{V z} \\
& \hline r^{2}
\end{aligned}
$$

onde $D$ - coeficiente de difusão molecular $\left(\mathrm{m}^{2} / \mathrm{s}\right) ; V_{Z}$ - velocidade axial de escoamento $(\mathrm{m} / \mathrm{s}) ; f$ - fator de eficiência do iniciador; $[P \bullet]$ - concentração de macromoléculas ativas $\left(\mathrm{kmol} / \mathrm{m}^{3}\right)$.

As equações obtidas para os momentos do polímero vivo de ordem zero $\left(\lambda_{0}\right)$, um $\left(\lambda_{1}\right)$ e dois $\left(\lambda_{2}\right)$ são apresentadas a seguir, respectivamente ${ }^{[20]}$ :

$$
\begin{aligned}
& \lambda_{0}=\left(\frac{2 f k_{d} C_{I}+2 k_{i t} C_{M}^{3}}{k_{t c}}\right)^{\frac{1}{2}} \\
& \lambda_{1}= \\
& \frac{2 f k_{d} C_{I}+2 k_{i t} C_{M}^{3}+\lambda_{0}\left(k_{p} C_{M}+k_{t r m} C_{M}+k_{t r s} C_{S}\right)}{k_{t r m} C_{M}+k_{t r s} C_{S}+k_{t c} \lambda_{0}}
\end{aligned}
$$

$\lambda_{2}=$

$$
\frac{2 f k_{d} C_{I}+2 k_{i t} C_{M}^{3}+k_{p} C_{M}\left(2 \lambda_{1}+\lambda_{0}\right)+\lambda_{0}\left(k_{t r m} C_{M}+k_{t r s} C_{S}\right)}{k_{t r m} C_{M}+k_{t r s} C_{S}+k_{t c} \lambda_{0}}
$$

Realizando um balanço de energia em um volume de controle do reator tubular e desenvolvendo os operadores matemáticos para um sistema em coordenadas cilíndricas, obteve-se a seguinte equação diferencial parcial para estimar a temperatura (T em Kelvin) ao longo do reator tubular ${ }^{[5,14,19,20]}$.

$$
\begin{aligned}
& \frac{\partial T}{\partial Z}= \\
& {\left[\frac{k}{\rho \cdot C p} \cdot\left(\frac{\partial^{2} T}{\partial r^{2}}+\frac{1}{r} \cdot \frac{\partial T}{\partial r}\right)-\frac{\Delta H}{\rho \cdot C p} \cdot k_{p} \cdot C_{m} \cdot[P \cdot]\right] \cdot \frac{1}{V z}}
\end{aligned}
$$

onde $k$ - condutividade térmica da mistura reacional $\left(\mathrm{J} / \mathrm{m}^{2} . \mathrm{s} . \mathrm{K}\right) ; C p$ - capacidade calorífica da mistura reacional $(\mathrm{J} / \mathrm{Kg} . \mathrm{K}) ; \rho$ - densidade da mistura reacional $\left(\mathrm{kg} / \mathrm{m}^{3}\right) ;$ e $\Delta H$ - calor de polimerização (J/kmol).

Aplicando as hipóteses mencionadas anteriormente nas equações da continuidade e Navier-Stokes, e considerando um fluxo mássico constante através do reator, obtêm-se, respectivamente, as seguintes equações para o cálculo da queda de pressão ${ }^{[14]}$ e da velocidade axial do escoamento ${ }^{[5,14,19,20]} \mathrm{em}$ qualquer posição radial ao longo do reator tubular:

$$
\begin{aligned}
& \left(\frac{\partial P}{\partial Z}\right)=-\frac{Q}{\pi \cdot \int_{0}^{R} \rho \cdot r \cdot \int_{R}^{r} \frac{r}{\mu} \cdot d r \cdot d r} \\
& V z=-\frac{\frac{Q}{2} \cdot{ }_{R}^{r} \frac{r}{\mu} \cdot d r}{\pi \cdot \int_{0}^{R} \rho \cdot r \int_{R}^{r} \frac{r}{\mu} \cdot d r \cdot d r}
\end{aligned}
$$

onde $P$ - pressão interna do reator tubular $\left(\mathrm{N} / \mathrm{m}^{2}\right) ; Q$ - vazão mássica através do reator tubular $(\mathrm{kg} / \mathrm{s}) ; \mu$ - viscosidade da mistura reacional (cP); e $R$ - raio total do reator tubular (m).

As condições de contorno no centro do reator, associadas à velocidade do escoamento $\left(\mathrm{V}_{\mathrm{z}}\right)$, ao balanço de massa, energia e momentos do polímero morto, são de derivada radial nula da propriedade em questão devido à simetria do reator. Já na parede, respectivamente, são de velocidade nula, de derivada radial nula da propriedade em questão para os balanços de massa e momentos do polímero morto, e de igualdade entre o calor transferido por convecção e por condução para o balanço de energia.

A resolução numérica do modelo matemático do reator tubular foi realizada em duas etapas. A primeira etapa consistiu na discretização dos termos radiais, inclusive nas condições de contorno, através da aplicação do Método da Colocação Ortogonal. Após a discretização dos termos radiais, o sistema resultante de equações diferenciais ordinárias não- 
lineares foi resolvido numericamente utilizando-se o método de integração implícita de Adams-Moulton. A característica mais importante deste método de integração é a sua capacidade de superar problemas de rigidez (stiffness), freqüentemente reportados nos estudos de modelagem e simulação de processos de polimerização ${ }^{[21-23]}$.

O Método dos Momentos foi empregado para obter um modelo de caracterização físico-química capaz de estimar os pesos moleculares médios numérico e ponderal do polímero, bem como o índice de polidispersão das cadeias poliméricas.

\section{Resultados e Discussão}

Na primeira parte deste estudo ${ }^{[7]}$, o efeito da pré-polimerização no comportamento dos reatores tubulares e nas propriedades do polímero obtido, foi analisado comparando-se os perfis radiais das variáveis de processo gerados em sistemas reacionais com apenas um ou dois estágios. Neste estudo preliminar, dois sistemas de polimerização com o mesmo volume reacional foram testados, sendo um com pré-polimerização (dois estágios) e o outro sem pré-polimerização, ou seja, apenas o reator tubular. Os resultados mostraram que a utilização de um CSTR como pré-polimerizador leva a uma redução significativa dos largos perfis radiais de todas as variáveis de processo avaliadas (velocidade axial, temperatura, conversão de monômero, viscosidade e densidade da massa reacional, peso molecular médio ponderal e numérico, além do índice de polidispersão do polímero), desenvolvidos ao longo do reator tubular. Assim, o uso de um CSTR como prépolimerizador resultou em um polímero com características moleculares mais homogêneas na saída do reator tubular quando o CSTR foi empregado como primeiro estágio.

Partindo destas premissas, o presente trabalho busca aprofundar ainda mais o estudo da influência do uso de CSTR's como pré-polimerizadores no comportamento de reatores tubulares. Nesta seção são apresentados os resultados da influência da alimentação de diferentes frações de polímero (diferentes valores de conversão de monômero) com características moleculares médias muito semelhantes, nos perfis radiais das principais variáveis de processo, desenvolvidos no interior do reator tubular. A variação da fração de polímero na alimentação do reator tubular foi feita alterando-se o tempo de residência no pré-polimerizador (CSTR). Para isto, situações considerando diferentes volumes para este equipamento $\left(\mathrm{V}_{\mathrm{CSTR}}\right)$ foram simuladas, mantendo-se o volume do reator tubular e os demais parâmetros de processo sempre constantes. Diferentes frações de um polímero de peso molecular moderado $\left(\mathrm{Mw}_{0}=113000 \pm 6000 \mathrm{~g} / \mathrm{mol}\right)$ foram alimentadas no reator tubular. Uma conversão na entrada deste equipamento $\left(\mathrm{X}_{0}\right)$ de $2,5 \%$ foi obtida no CSTR de 1,0 litro de volume, $X_{0}=11,0 \%$ em um CSTR de 5,0 litros, $X_{0}=19,0 \%$ em um CSTR de 10,0 litros, $X_{0}=30,0 \%$ em um CSTR de 20,0 litros e $\mathrm{X}_{0}=37,0 \%$ em um CSTR de 30,0 litros. As seguintes condições operacionais e dimensões do reator tubular foram consideradas nas simulações: $\mathrm{L}=200,0 \mathrm{~m}$ (compri- mento do reator tubular); $\mathrm{Di}=0,0254 \mathrm{~m}$ (diâmetro interno do reator tubular); $\mathrm{Q}=0,0005 \mathrm{~kg} / \mathrm{s}$ (vazão mássica através do reator tubular); $\mathrm{Ci}_{0}=0,012 \mathrm{~mol} / \mathrm{l}$ (concentração inicial de iniciador); $\mathrm{T}_{\mathrm{pol}}=343,15 \mathrm{~K}$ (temperatura de polimerização); $\mathrm{T}_{\mathrm{c}}=343,15 \mathrm{~K}$ (temperatura da camisa do reator tubular); $\varphi_{\mathrm{s}}=0,20$ (fração volumétrica de solvente). Estas condições operacionais, e especificamente a baixa vazão mássica (longos tempos de residência), foram selecionadas para permitir uma avaliação da completa evolução dos perfis desenvolvidos no interior do reator tubular.

De acordo com os resultados obtidos, quanto maior a fração de polímero e, conseqüentemente, maior a viscosidade da mistura reacional alimentada no reator tubular, menos acentuados são os perfis radiais de todas as variáveis de processo, desenvolvidos no interior deste equipamento.

A Figura 1 permite analisar o efeito da fração de polímero alimentada no reator tubular sobre a velocidade axial do escoamento no centro do reator tubular, pois trata-se da região onde aparecem as maiores variações deste parâmetro de processo.

Observando a Figura 1, nota-se uma maior aceleração da massa reacional, com posterior oscilação, quando baixos volumes de CSTR foram testados, ou seja, quando baixas frações poliméricas foram alimentadas ao reator tubular $\left(\mathrm{V}_{\text {CSTR }}=1,0\right.$ litro $\left.\rightarrow \mathrm{X}_{0}=2,5 \%\right)$. O brusco aumento da velocidade axial do escoamento verificado na região central do reator se deve às diferenças na variação da viscosidade nesta região e na parede. À medida que a viscosidade na parede aumenta, há uma redução na velocidade de escoamento nesta região que, em virtude da consideração matemática de fluxo mássico constante ao longo da seção transversal, deve ser compensada com um aumento da velocidade na região central do equipamento. Com isso, observam-se fortes distorções do perfil radial da velocidade ao longo do equipamento e, consequentemente, nos perfis radiais de todas as outras variáveis de processo.

Com o aumento da fração de polímero na corrente de alimentação $\left(\mathrm{V}_{\mathrm{CSTR}}=30,0\right.$ litros $\left.\rightarrow \mathrm{X}_{0}=37,0 \%\right)$, nota-se uma considerável diminuição da velocidade máxima no centro do reator, o que leva a uma conseqüente redução das oscilações

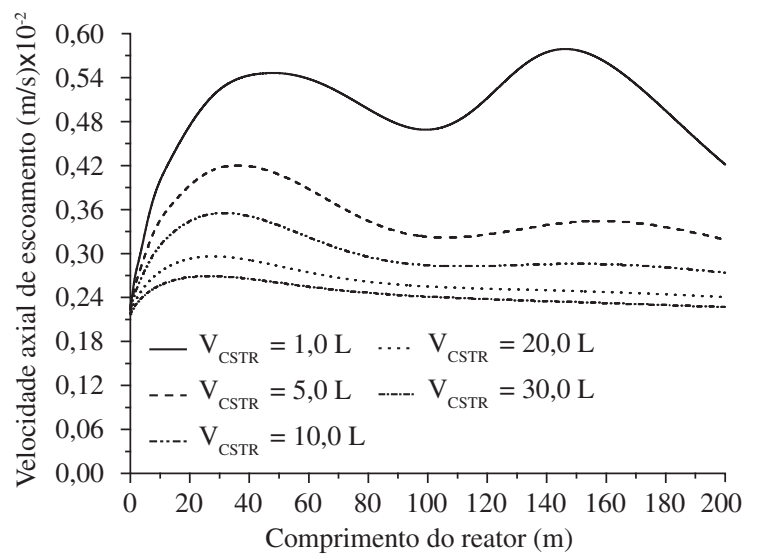

Figura 1. Perfil da velocidade axial de escoamento no centro do reator tubular para diferentes frações de polímero em sua alimentação. 
dos perfis axiais da velocidade axial e suavização das distorções dos perfis radiais de todas as variáveis de processo, principalmente quando volumes de CSTR acima de 10,0 litros foram testados. Assim, é possível afirmar que o aumento da fração de polímero alimentada leva a um estreitamento da distribuição do tempo de residência das macromoléculas no reator tubular, resultando em um polímero com propriedades finais mais homogêneas.

Pelo fato da temperatura da reação apresentar as maiores variações na região central do reator tubular, os resultados para esta variável também são apresentados apenas no centro do equipamento. A Figura 2 mostra que, quanto maior o valor da conversão de monômero alimentada no reator, menor deve ser a máxima temperatura atingida no equipamento.

Portanto, além da redução da geração de calor pela reação, o que é plenamente favorável do ponto de vista de controle das qualidades do polímero produzido, o aumento da fração de polímero na alimentação do reator tubular também reduz as instabilidades no escoamento da massa reacional ao longo deste equipamento. Isto permite que o reator seja operado mais facilmente e de forma muito mais segura, reduzindo as possibilidades de canalização do fluxo (flow channeling), entupimento do equipamento (fouling), surgimento de pontos quentes (hot spots) e ocorrência de disparos térmicos não controlados da reação (thermal runway).

A Figura 3 apresenta a evolução dos perfis axiais e radiais do peso molecular médio ponderal do polímero quando uma massa reacional com viscosidade baixa (Figura 3a) e outra com uma viscosidade moderada (Figura $3 b$ ) foram alimentadas no reator tubular. Como se pode observar, o aumento da fração de polímero alimentada reduziu consideravelmente os perfis radiais gerados ao longo do equipamento. Na saída do equipamento nota-se que, na primeira situação simulada (baixa viscosidade alimentada), a distribuição dos valores de peso molecular na direção radial é mais heterogênea do que a observada na segunda situação. Assim, o aumento da fração de polímero, conseguida com o aumento do tempo de residência no pré-polimerizador (CSTR), deve resultar no estreitamento da distribuição do tempo de residência das ma-

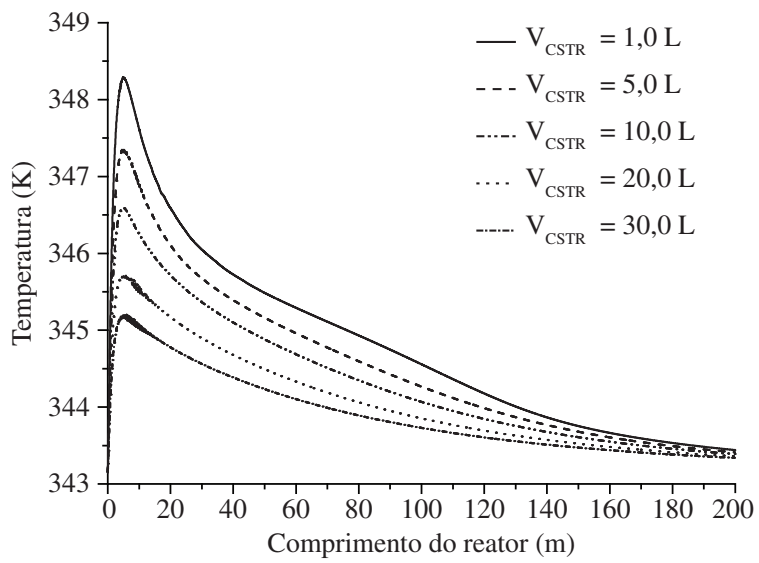

Figura 2. Perfil da temperatura no centro do reator tubular para diferentes frações de polímero em sua alimentação.

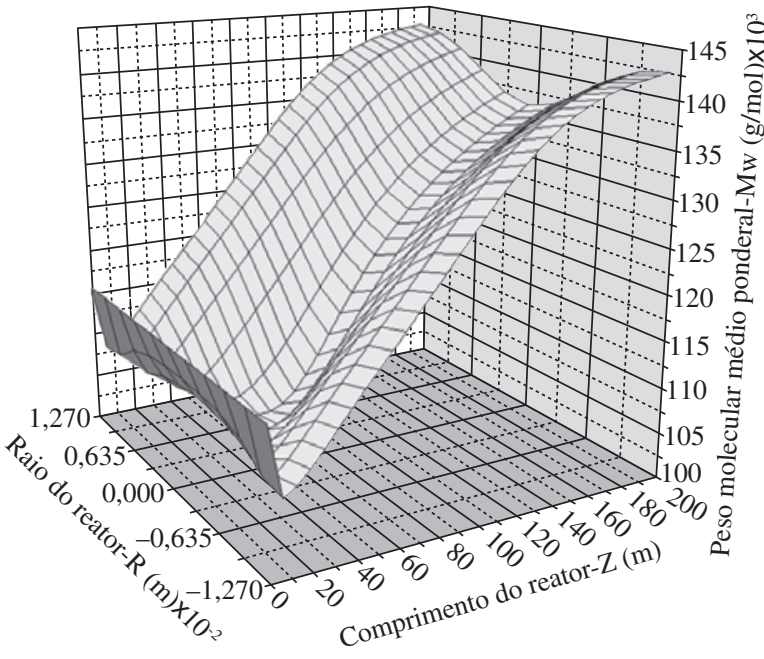

(a)

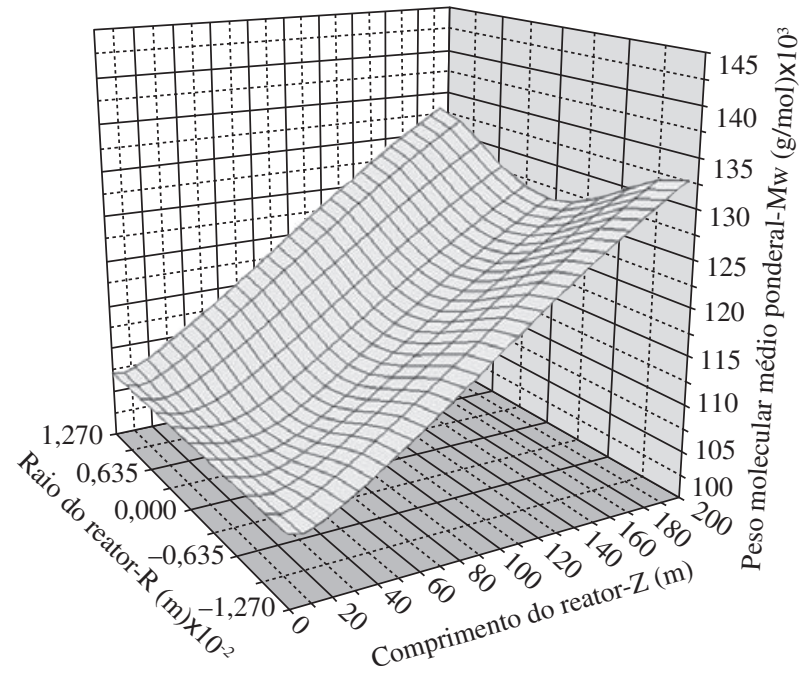

(b)

Figura 3. Perfil do peso molecular médio ponderal do polímero no reator tubular para diferentes frações de polímero em sua alimentação: a) $\mathrm{V}_{\text {CSTR }}=$ 5,0 litros $\rightarrow X_{0}=11 \%$; b) $\mathrm{V}_{\text {CSTR }}=30,0$ litros ou $X_{0}=37 \%$.

cromoléculas no reator tubular, e fornecendo um polímero com propriedades mais homogêneas.

Para permitir uma melhor avaliação da influência da alimentação de diferentes frações de polímero no comportamento do reator tubular, os valores médios das variáveis de interesse foram calculados ao longo do equipamento, conforme a equação genérica abaixo:

$$
\bar{\psi}=\frac{1}{A} \iint_{A} \psi d A=\frac{2}{R^{2}} \int_{0}^{R} \psi r d r
$$

onde A - área de seção transversal do reator tubular $\left(\mathrm{m}^{2}\right)$

Calculando a velocidade média do escoamento através do reator tubular, verificou-se que, independentemente da viscosidade da massa reacional alimentada neste equipamento, os perfis gerados são muito semelhantes e a velocidade média permanece praticamente constante durante todo o escoamento. Por este motivo, este resultado não é mostrado no presente artigo. 
A Figura 4 apresenta os perfis axiais dos valores médios das propriedades moleculares do polímero produzido (Figuras $4 \mathrm{a}$ e b) e da taxa de conversão de monômero (Figura 4c) ao longo do reator tubular. Os resultados mostram que, apesar da grande diferença entre os perfis desenvolvidos neste equipamento, obteve-se um polímero com propriedades moleculares muito semelhantes em todas as situações simuladas (Figuras 4a e b). Porém, o aumento da viscosidade da massa

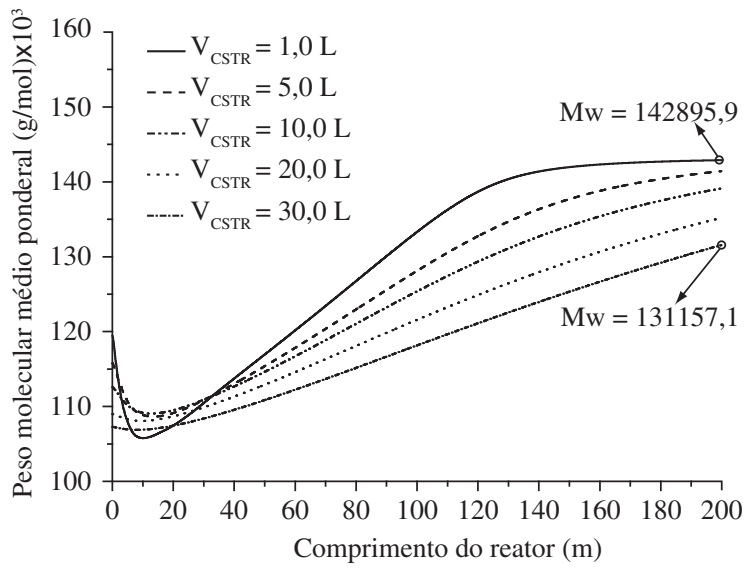

(a)

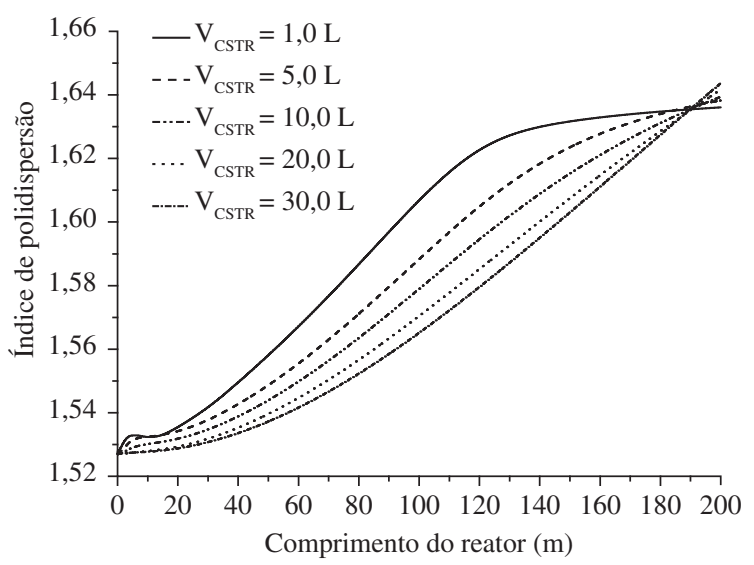

(b)

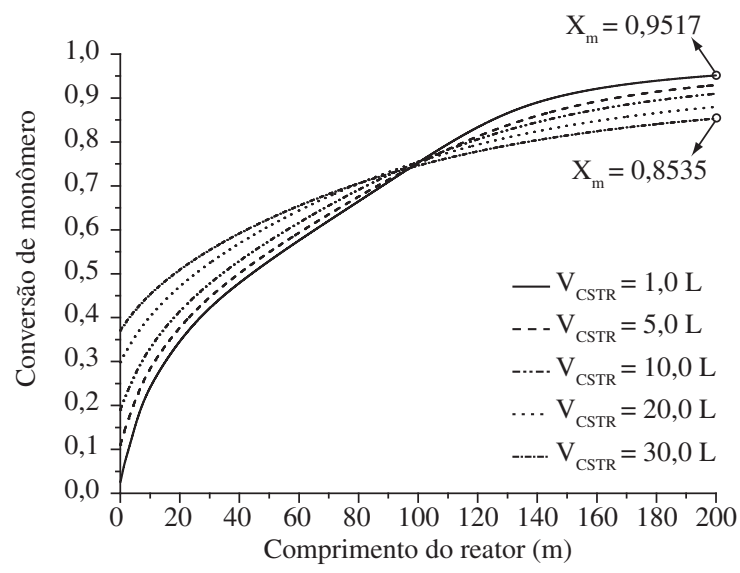

(c)

Figura 4. Perfil axial médio para as condições simuladas: a) peso molecular médio ponderal do polímero; b) índice de polidispersão do polímero; e c) conversão de monômero. reacional alimentada ao reator tubular resultou em menores valores de conversão na saída do equipamento (Figura 4c), principalmente porque uma menor quantidade de calor é liberada pela reação (Figura 2) nestas situações. Outro motivo é o característico rendimento inferior apresentado pelos CSTR's quando comparados aos reatores tubulares. Sendo assim, quanto mais tempo a mistura reacional permanecer no interior do CSTR, menor deve ser a conversão obtida na saída do reator tubular.

\section{Conclusões}

Os resultados mostram que, com a utilização de um CSTR como pré-polimerizador, se a fração de polímero na alimentação do reator tubular for aumentada, é possível reduzir significativamente os perfis radiais desenvolvidos ao longo deste equipamento, fornecendo um polímero com propriedades mais homogêneas. $\mathrm{O}$ aumento da fração de polímero na entrada do reator tubular contribui com a diminuição do calor gerado pela reação no interior deste equipamento, o que é plenamente favorável do ponto de vista de controle das qualidades do polímero produzido. Além disso, o aumento da fração de polímero é capaz de eliminar possíveis instabilidades no escoamento da massa reacional ao longo deste equipamento. Isto permite que o reator seja operado mais facilmente e de forma muito mais segura, minimizando a possibilidade de ocorrência de fenômenos indesejáveis, como a canalização do fluxo (flow channeling), o entupimento do equipamento (fouling), o surgimento de pontos quentes (hot spots) e a ocorrência de disparos térmicos não controlados da reação (thermal runway).

Portanto, como o modelo matemático desenvolvido neste estudo provou ser capaz de prever a ocorrência dos indesejáveis fenômenos mencionados anteriormente, é possível utilizá-lo na estimativa de condições operacionais ótimas e seguras que forneçam um polímero com propriedades específicas para aplicações de interesse.

\section{Referências Bibliográficas}

1. McGreavy, C. - "Polymer Reactor Engineering", $1^{\text {st }}$ ed., VCH Publishers, New York (1994);

2. Cabral, P. A.; Melo, P. A.; Biscaia Jr., E. C.; Lima, E. L. \& Pinto, J. C. - Polym. Eng. Sci., 43, 6, p.1163 (2003);

3. Tien, N.-K.; Flaschel, E. \& Renken, A. - "Polymer Reaction Engineering - Influence of Reaction Engineering on Polymer Properties", Hanser Publishers (1983);

4. Chen, C. C. - Polym. Plast. Technol. Eng., 33, 1, p.55 (1994);

5. Lynn, S. \& Huff, J. E. - AIChe J., 17, 2, p.475 (1971);

6. Husain, A. \& Hamielec, A. E. - AIChe Symp. Series, Vol. 72, 160, p.112 (1976);

7. Nogueira, A. L.; Lona, L. M. F. \& Machado, R. A. F. - J. Appl. Pol. Sci., 91, p.871 (2004); 
8. Soroush, M \& Kravaris, C. - AIChe J., 39, p.1920 (1993);

9. Marten, F. L. \& Hamielec, A. E. - J. Appl. Pol. Sci., 27, p.489 (1982);

10. Stevens, C. J. - "Mathematical Modeling of Bulk and Solution Free Radical Polymerization in Tubular Reactors", Ph.D. Thesis, University of Winscosin, Madison - EUA (1988);

11. Hui, A. \& Hamielec, A. E. - J. Pol. Sci., 25, p.167 (1968);

12. Hamer, J. W. \& Ray, W. H. - Chem. Eng. Sci., 41, 12, p.3083 (1986);

13. Holman, J. P. - "Heat Transfer", Mc Graw Hill, New York (1963);

14. Chen, C. C. \& Nauman, E. B. - Chem. Eng. Sci., 44, 1, p.179 (1989);

15. Cutter, L. A. \& Drexler, T. D. - Comp. Appl. Pol. Sci., p.13 (1982);
16. Hui, A. \& Hamielec, A. E. - J. Appl. Pol. Sci., 16, p.749 (1972);

17. Husain, A. \& Hamielec, A. E. - J. Appl. Pol. Sci., 22, p.1207 (1978);

18. Kricheldorf, H. R. - "Handbook of Polymer Synthesis", Marcel Dekker, New York (1992);

19. McLaughlin, H. S.; Mallikarjun, R. \& Nauman, E. B. - AIChe J., 32, 3, p. 419 (1986);

20. Wyman, C. E.; Carter, L. F. - AIChe Symp. Ser., 72, 160, p.1 (1976);

21. Powell, F. E. \& Brooks, B. W. - Chem. Eng. Sci., 50, 5, p.837 (1995);

22. Soliman, M. A.; Aljarboa, T. \& Alahmad, M. - Polym. Eng. Sci., 34, p.1464 (1994);

23. Tossun, G. - AIChe J., 38, p.425 (1992).

Enviado:09/11/06

Reenviado: 08/05/07

Aceito: 11/05/07 